\title{
Hirschsprung's Disease Complicated by Sigmoid Volvulus: A Systematic Review
}

\author{
Ufuk Uylas (D), Orgun Gunes $\mathbb{1}$, Cuneyt Kayaalp
}

Department of Gastroenterology Surgery, İnönü University School of Medicine, Malatya, Turkey

Background: Hirschsprung's disease and sigmoid volvulus can sometimes be seen in the same patient.

Aims: To investigate the presence of Hirschsprung's disease in patients with sigmoid volvulus and to discuss the diagnosis and treatment methods.

Study Design: Systematic review.

Methods: This systematic review has been reported in line with the Preferred Reporting Items for Systematic Reviews and Meta-Analyses and the methodological quality of systematic reviews guidelines. The PubMed and Scopus databases were scanned using the keywords "Hirschsprung* volvulus*" and "congenital aganglionic megacolon volvulus*". The reference list of the selected studies was reviewed for cross-checking. Two reviewers independently screened the available literature. Only the Hirschsprung's disease cases involving sigmoid volvulus were included, and cases of patients with volvulus in other sites was excluded. There was no restriction with respect to the publication language and type of writing. The primary outcome was morbidity and mortality.

Results: A total of 31 cases were analyzed in 22 articles; $97 \%$ of the patients were under the age of $40,90 \%$ were male. There was a statistically significant difference in the necessity for relaparotomy between patients who were scheduled for sigmoid volvulus therapy with the suspicion of Hirschsprung's disease and patients who were treated without suspicion of Hirschsprung's disease ( $0 \%$ vs $37.5 \%, p=0.02)$. While there was no postoperative death in cases with suspected Hirschsprung disease, this mortality rate was $25 \%$ in cases without suspicion ( $p=$ 0.08).

Conclusion: Hirschsprung's disease should be excluded with rectal biopsy if a patient with sigmoid volvulus is under 40 years of age and has complaints of constipation from childhood.
Hirschsprung's disease and sigmoid volvulus are bowel diseases that are accompanied by chronic constipation. Both are more common in men (1). Hirschsprung's disease is more prevalent in newborns and in childhood, but a less aggressive form of a short-segment Hirschsprung's disease can be diagnosed in adolescence or in adulthood (2). Sigmoid volvulus is seen in patients at older ages, and it is rarely encountered in children (3). Sometimes both pathologies may occur in the same individual. Hirschsprung's disease is a congenital disease, and sigmoid volvulus is a complication of Hirschsprung's disease. It is important to diagnose an underlying Hirschsprung's disease in sigmoid volvulus cases.

The basis of the treatment of Hirschsprung's disease is resection of the aganglionic segment and construction of coloanal anastomosis between the normal colon and anal canal. To achieve this, various treatment modalities have been developed (4). The management approach of sigmoid volvulus includes derotation of the sigmoid colon by endoscopic, enema, or surgical approaches in patients with viable bowel. After this step, elective surgery can be planned for patients with acceptable anesthesia and operative risk. In patients with a failed derotation attempt, findings of acute abdomen, or a gangrenous bowel, emergency surgery is indicated $(5,6)$. If the associated Hirschsprung's disease is overlooked, the outcomes of the treatment of sigmoid volvulus alone are not clear. This study aimed to provide clarity with a comprehensive systematic review of the literature.

\section{MATERIALS AND METHODS}

This systematic review was prepared on the basis of the Preferred Reporting Items for Systematic Reviews and Meta-Analyses.

\section{Search strategy}

The PubMed and Scopus databases were scanned using the keywords "Hirschsprung* volvulus*" and "congenital aganglionic megacolon volvulus*". The final screening took place on May 13,

Address for Correspondence: Ufuk Uylas, Department of Gastroenterology Surgery, İnönü University School of Medicine, Malatya, Turkey

e-mail: ufukuylas@hotmail.com

Received: April 20, 2020 Accepted: August 26, 2020 •DOI: 10.4274/balkanmedj.galenos.2020.2020.4.131

Available at www.balkanmedicaljournal.org

ORCID iDs of the authors: U.U. 0000-0003-4195-5498; Ö.G. 0000-0002-0576-6086; C.K. 0000-0003-4657-2998.

Cite this article as:

Uylas U, Gunes O, Kayaalp C. Hirschsprung's Disease Complicated by Sigmoid Volvulus: A Systematic Review. Balkan Med J 2021;38:1-6

Copyright@Author(s) - Available online at http://balkanmedicaljournal.org/ 
2020. The reference list of the selected studies was reviewed for cross-checking.

\section{Selection criteria and intervention(s)}

There was no restriction with respect to the publication language and type of articles. Only the Hirschsprung's disease cases involving sigmoid volvulus were included, and other volvulus cases (cecal, transverse, or splenic) were excluded. An e-mail was sent to the authors to obtain the missing data (such as surgical outcome, gender, and age) in their articles.

\section{Data extraction and study quality}

The methodological quality of the systematic reviews was used to assess the quality of this review (7). The Cochrane Handbook for Systematic Reviews of Interventions (the Handbook) was used for evaluating the risk of bias of the included studies in this review (8). This systematic review was registered in an international database (International Prospective Register of Systematic Reviews, CRD42020166692). Most studies reporting concomitant Hirschsprung's disease and sigmoid volvulus mainly include isolated case reports or small number of case series because this entity is rare. The year of publication, country of publication, age, sex, the presence of gangrene, surgical or nonsurgical treatments, and outcomes were reviewed. The data were evaluated separately by 2 researchers (UU and CK). A total of 31 cases were included in the analysis in 22 studies that met the defined inclusion criteria (Figure 1). Patients were grouped according to the following age groups: 0 to 2 years, newborn and infant; 2 to 17 years, pediatric; and $\geq 18$ years, adults.

\section{Statistical analysis}

Statistical analyses were performed using the Statistical Package for the Social Sciences Version 22.0 (IBM SPSS Corp, Armonk, NY, USA). Quantitative variables were expressed as mean \pm standard deviation (SD), median, minimum-maximum, and interval. Qualita-
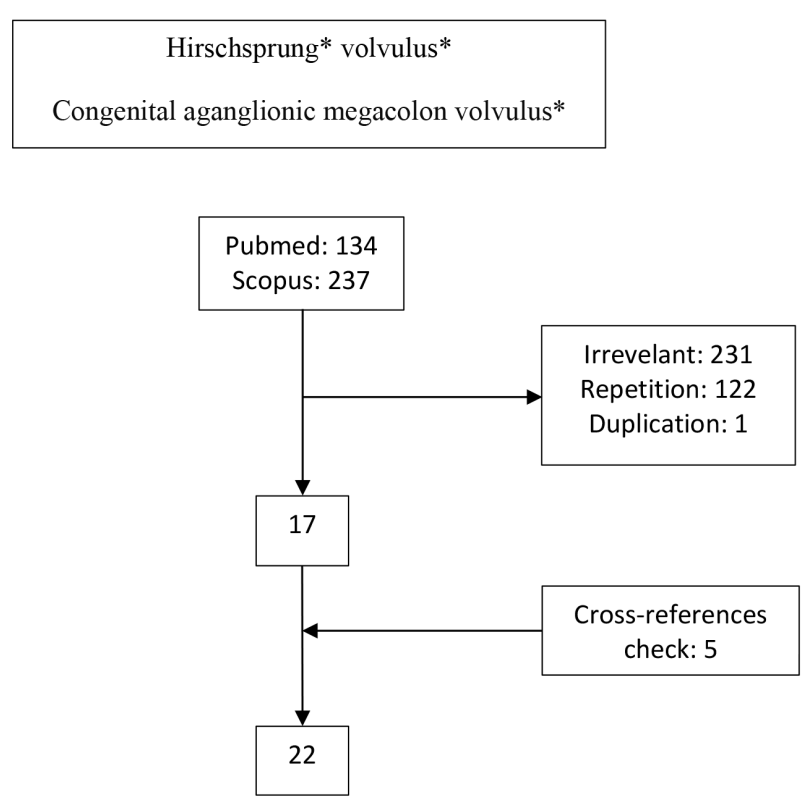

FIG. 1. Flowchart of the systematic review. tive variables were reported as numbers and percentages. The Shapiro-Wilk test was used to assess the normality distribution of the quantitative variables. Although the means and SDs were used for homogeneous distributions, median and range were used for heterogeneous distributions. The Fisher's Chi-square test was used to compare the qualitative variables. The Mann-Whitney $U$ test was used for heterogeneous distributions, and the Student's $t$ test was used for homogeneous distributions. $\mathrm{p}<0.05$ was considered statistically significant.

\section{RESULTS}

The selected publications included 16 cases from Asia, 8 cases from Europe, 4 cases from Africa, and 3 cases from North America (9-26). The age distribution of the patients was not homogeneous (Shapiro-Wilk test, $\mathrm{p}<0.05$ ). The age range was 4 hours to 82 years (median=10 years). Median age for newborn/infant, child, and adult was 1 day (range, 1-42 days), 7 years (range, 3-12 years), and 24 years (range, 18-82 years), respectively; 97\% of the patients were younger than 40 years and $90 \%$ were men (Table 1 ).

Nonoperative detorsion was attempted in 14 of 25 patients (56\%) ( 7 with enema, 2 with rectal tube, and 5 with endoscopy), with a success rate of $64.3 \%(9 / 14)$. A total of 11 patients (failed nonoperative derotation in 5 patients; nonoperative intervention 6 patients was not attempted) underwent surgical detorsion.

In $19(70.4 \%)$ patients, Hirschsprung's disease was diagnosed or suspected during the treatment of sigmoid volvulus (Table 2). In these 19 patients, the treatment of Hirschsprung's disease was successfully performed in a single procedure or stepwise surgical intervention. In the remaining 8 patients in whom the Hirschsprung's disease diagnosis was overlooked, 3 patients required early relaparotomy after the first operation, and 2 of these patients died. There was a difference in the necessity for relaparotomy $(37.5 \%$ vs $0 \%, p=0.02$ ) between patients who were scheduled for sigmoid volvulus therapy with the suspicion of Hirschsprung's disease and patients who were treated without suspicion of Hirschsprung's disease. Although mortality was not observed in 19 patients who were suspected to have Hirschsprung's disease, mortality was observed in 2 of the 8 patients who received only sigmoid volvulus treatment without considering Hirschsprung's disease ( $25 \%$ vs $0 \%, \mathrm{p}=0.08)$. In 4 patients, there were no data on this issue.

Intraoperative bowel gangrene was observed in only $3(9.7 \%)$ patients. In 1 patient, gangrene was observed in the entire colon. The surgical procedures performed for Hirschsprung's disease showed heterogeneity. The Soave procedure was performed in 6 patients, Swenson procedure in 11 patients, sphincterotomy in 1 patient, and restorative proctocolectomy in 1 patient.

Mortality was observed in a total of $2(8 \%)$ patients, where 1 was of pediatric age and the other was an adult. Both the patients did not have gangrene. However, neither of the patients had undergone definitive surgery for Hirschsprung's disease because of unawareness of a Hirschsprung's disease diagnosis. One of these patients underwent only surgical detorsion, and the cause of death was unknown. Resection and anastomosis with transverse colostomy were performed in the other patient. After the closure of the colostomy, relaparotomy was necessary owing to massive abdominal 
TABLE 1. Reports of 31 Hirschsprung's diseases complicated by sigmoid volvulus in chronological order

\begin{tabular}{|c|c|c|c|c|c|}
\hline Author & Country & Year & Age & Gender & Perioperative findings \\
\hline Dean (9) & United States of America & 1952 & 4 years & Male & SV \\
\hline Shepherd $\left(10^{\mathrm{a}}\right)$ & Uganda & 1969 & 12 years & Male & SV \\
\hline Ciardimi et al. $\left(10^{\mathrm{a}}\right)$ & Italy & 1977 & 3 years & Male & SV \\
\hline Ciardimi et al. $\left(10^{\mathrm{a}}\right)$ & Italy & 1977 & 5 years & Male & SV \\
\hline Buts et al. (11) & Belgium & 1980 & 10 years & Female & SV \\
\hline Valla et al. (10a) & France & 1982 & 5 days & Male & SV \\
\hline Valla et al. (10a) & France & 1982 & 5 years & Male & SV \\
\hline McCalla et al. (12) & United States of America & 1985 & 2 days & Male & SV \\
\hline Henales et al. $\left(10^{\mathrm{a}}\right)$ & Spain & 1993 & 5 years & Male & SV \\
\hline Erdener et al. (13) & Turkey & 1995 & 3 years & Male & SV \\
\hline Venugopal et al. (14) & United Kingdom & 1997 & 4 hours & Male & SV \\
\hline Sarioglu et al. (15) & Turkey & 1997 & 11 years & Male & SV \\
\hline Rachid (16) & Morocco & 1997 & Adult $^{\mathrm{b}}$ & Male $^{\mathrm{b}}$ & SV \\
\hline Puneet et al. (17) & India & 2000 & 12 years & Male & Gangrenous SV \\
\hline Bach et al. (18) & Malawi & 2003 & Adult $^{\mathrm{c}}$ & N/A & SV \\
\hline Tan et al. (19) & Singapore & 2006 & 38 years & Male & Gangrenous $\mathrm{SV}^{\mathrm{d}}$ \\
\hline Ghaemi et al. (20) & Iran & 2010 & 18 years & Female & SV \\
\hline Ghaemi et al. (20) & Iran & 2010 & 21 years & Male & SV \\
\hline Ghaemi et al. (20) & Iran & 2010 & 19 years & Male & SV \\
\hline Ghaemi et al. (20) & Iran & 2010 & 24 years & Male & SV \\
\hline Alagumuthu et al. (21) & India & 2011 & 33 years & Male & SV \\
\hline Alagumuthu et al. (21) & India & 2011 & 18 years & Female & SV \\
\hline Alagumuthu et al. (21) & India & 2011 & 24 years & Male & SV \\
\hline Zeng et al. (10) & United States of America & 2012 & 12 years & Male & SV \\
\hline Ibrahim et al. (22) & Egypt & 2013 & 7 years & Male & SV \\
\hline Khalayleh et al. (23) & Israel & 2016 & 1.25 days & Male & SV \\
\hline Khalayleh et al. (23) & Israel & 2016 & 1.25 days & Male & SV \\
\hline Khalayleh et al. (23) & Israel & 2016 & 1 days & Male & SV \\
\hline Ranjan et al. (24) & India & 2016 & 11 years & Male & SV \\
\hline Van Horebeek et al. (25) & Belgium & 2017 & 42 days & Malee & SV \\
\hline $\mathrm{Wu}(26)$ & China & 2018 & 82 years & Male & Gangrenous SV \\
\hline
\end{tabular}

N/A: not applicable, SV: sigmoid volvulus

aReference found in cited review article; bFrom Scopus; cAdulthood of the patient was understood from the picture in the article; dGangrenous sigmoid colon, duskiness involving the entire colon; eThe author of the study reported by e-mail.

distension. There was anastomotic leakage, and primary repair and a transverse colostomy were performed. Hirschsprung's disease was detected in the rectal biopsy, but the patient died from the septic shock of fecal peritonitis. No mortality was seen in patients undergoing a definitive surgery for Hirschsprung's disease.

\section{DISCUSSION}

The incidence of Hirschsprung's disease is approximately 1 in 5000 live births $(27,28)$, which may change among ethnic groups. The incidence of Hirschsprung's disease is 1.5 in 10,000 live births in Northern Europe and 2.1 in African American and 2.8 in Asian populations (29). In this analysis, most patients with sigmoid volvulus had combined Hirschsprung's disease, 50\% of the cases $(15 / 30)$ were reported from Asia, and the lowest rate was from the United States, with $10 \%$ of the cases (3/30). The incidence of Hirschsprung's disease also varies between sexes. The male-to-female ratio is approximately $4: 1(1,30)$. This study found a similarly high incidence of the disease in men (90\%).

Approximately 95\% of patients with Hirschsprung's disease are diagnosed before the age of 5 years (31). Mild cases can be diagnosed after the age of 10 years and are called adult Hirschsprung's disease (32-34). The actual incidence of patients with adult Hirschsprung's 
TABLE 2. Reports of surgical approach for 31 cases of Hirschsprung's disease complicated by sigmoid volvulus in a chronological order

\begin{tabular}{|c|c|c|c|c|c|}
\hline Author & Derotation & Biopsy & Surgery & $\begin{array}{l}\text { Doubt on Hirschsprung's } \\
\text { disease }\end{array}$ & Outcome \\
\hline Dean (9) & Enema/yes & N/A & Surgical details are not available & N/A & Recovered \\
\hline Shepherd $\left(10^{a}\right)$ & Surgery/yes & N/A & Only derotation & No & Died \\
\hline Ciardimi et al. $\left(10^{\mathrm{a}}\right)$ & N/A & N/A & Surgical details are not available & N/A & N/A \\
\hline Ciardimi et al. $\left(10^{\mathrm{a}}\right)$ & N/A & N/A & Surgical details are not available & N/A & N/A \\
\hline Buts et al. (11) & Enema/failed & Yes & First sigmoidopexy, later Swenson & No & Recovered \\
\hline Valla et al. $\left(10^{\mathrm{a}}\right)$ & Surgery/yes & N/A & Only sigmoidectomy & No & Recovered \\
\hline Valla et al. $\left(10^{\mathrm{a}}\right)$ & Surgery/yes & N/A & Only sigmoidectomy & No & Recovered \\
\hline McCalla et al. (12) & Surgery/yes & Yes & $\begin{array}{c}\text { First only derotation, relaparotomy } y^{\mathrm{b}} \text {, later } \\
\text { Soave }\end{array}$ & No & Recovered \\
\hline Henales et al. $\left(10^{\mathrm{a}}\right)$ & Surgery/yes & N/A & Enterostomy and sphincterotomy & Yes & Recovered \\
\hline Erdener et al. (13) & Surgery/yes & Yes & First colostomy later Soave & Yes & Recovered \\
\hline Venugopal et al. (14) & Enema/yes & Yes & Soave & Yes & Recovered \\
\hline Sarioglu et al. (15) & Surgical/yes & Yes & First colostomy later Swenson & Yes & Recovered \\
\hline Rachid (16) & N/A & N/A & Surgical details are not available & N/A & N/A \\
\hline Puneet et al. (17) & N/A & Yes & Hartmann's procedure & No & Recovered \\
\hline Bach et al. (18) & N/A & Yes & $\begin{array}{l}\text { First mesosigmoidoplasty, then } \\
\text { relaparotomy }^{\mathrm{c}}\end{array}$ & No & Recovered \\
\hline Tan et al. (19) & Surgical/yes & Yes & Restorative proctocolectomy & Yes & Recovered \\
\hline Ghaemi et al. (20) & Endoscopy/yes & Yes & Swenson & Yes & N/A \\
\hline Ghaemi et al. (20) & Endoscopy/failed & Yes & First derotation, later Swenson & Yes & N/A \\
\hline Ghaemi et al. (20) & Endoscopy/failed & Yes & First derotation, later Swenson & Yes & N/A \\
\hline Ghaemi et al. (20) & Endoscopy/failed & Yes & First derotation, later Swenson & Yes & N/A \\
\hline Alagumuthu et al. (21) & Surgical/yes & Yes $^{\mathrm{d}}$ & $\begin{array}{l}\text { Anastomosis and colostomy } \mathrm{e}^{\mathrm{e}} \text { and } \\
\text { relaparotomy }\end{array}$ & No & Died \\
\hline Alagumuthu et al. (21) & Surgical/yes & Yes & First colostomy, ${ }^{\mathrm{f}}$ later Swenson & Yes & Recovered \\
\hline Alagumuthu et al. (21) & Rectal tube/yes & Yes & Soave $^{\mathrm{g}}$ & Yes & Recovered \\
\hline Zeng et al. (10) & Rectal tube/yes & Yes & Surgical details are not available & Yes & Recovered \\
\hline Ibrahim et al. (22) & Enema/failed & Yes & First colostomy, later Soave ${ }^{\mathrm{g}}$ & Yes & Recovered \\
\hline Khalayleh et al. (23) & Enema/yes ${ }^{\mathrm{h}}$ & Yes & Swenson & Yes & Recovered \\
\hline Khalayleh et al. (23) & Enema/yes & Yes & Swenson & Yes & Recovered \\
\hline Khalayleh et al. (23) & Enema/yes & Yes & Swenson & Yes & Recovered \\
\hline Ranjan et al. (24) & $\mathrm{N} / \mathrm{A}$ & $\mathrm{N} / \mathrm{A}$ & Soave $^{\mathrm{i}}$ & Yes & Recovered \\
\hline Van Horebeek et al. (25) & Endoscopy/yes & Yes & Swenson ${ }^{\mathrm{j}}$ & Yes & Recovered \\
\hline $\mathrm{Wu}(26)$ & Surgical/yes & Yes & First Hartmann's procedure later none & Yes & Recovered \\
\hline
\end{tabular}

N/A: not applicable

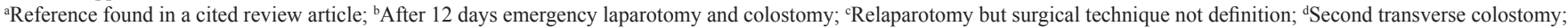

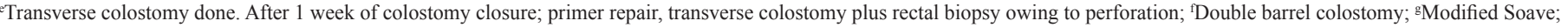

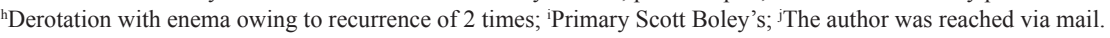

disease is unknown because Hirschsprung's disease is often overlooked in adult patients. There are cases of Hirschsprung's disease even at the ages of 74 and 82 years $(26,35)$. Approximately half of the adult patients with Hirschsprung's disease are younger than 30 years (2). In our study, $93 \%$ of the patients were younger than 30 years and $97 \%$ were younger than 40 years.

Hirschsprung's disease is seen in $0.6 \%$ to $3 \%$ of sigmoid volvulus cases $(15,23)$. Hirschsprung's disease frequency increases to $17 \%$ in the sigmoid volvulus cases below the age of 18 years (3). In a study by Ghaemi et al. (20), Hirschsprung's disease was detected in approximately 1 of 3 patients with sigmoid volvulus whose ages ranged between 14 and 30 years. The aganglionic segment in Hirschsprung's disease starts from the distal rectum, and the extent of the proximal diseased part varies. In $80 \%$ of individuals, aganglionosis is restricted to the rectosigmoid colon (short-segment disease); in $15 \%$ to $20 \%$ of patients, aganglionosis extends proximally to the sigmoid colon (long-segment disease); and in approximately 5\%, aganglionosis affects the entire large intestine (total 


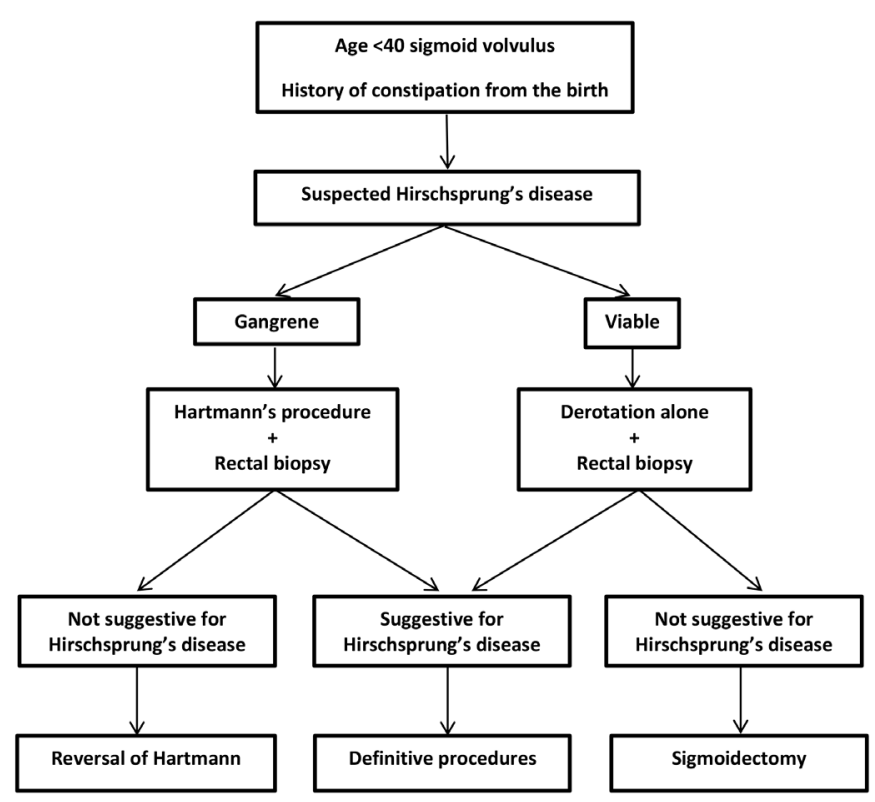

FIG. 2. Management of Hirschsprung's disease complicated by sigmoid volvulus.

colonic aganglionosis). Rarely, the aganglionosis extends into the small bowel or even more proximally to encompass the entire bowel (total intestinal aganglionosis). In most cases of late-diagnosed Hirschsprung's disease, the short segment of the bowel is affected. In these patients, prolonged constipation increases the likelihood of developing sigmoid volvulus (27).

Rectal biopsy for the diagnosis of Hirschsprung's disease is a worldwide accepted entity. Rectal biopsy is recommended from 1 to $3 \mathrm{~cm}$ above the dentate line (36). Although it is frequently used as a result of consensus, routine use of anorectal manometry or barium enema in Hirschsprung's disease is not necessary (37). Anorectal manometry has been reported to be more useful for neurogenic achalasia of the internal anal sphincter rather than for Hirschsprung's disease (37). It is difficult to detect the presence of Hirschsprung's disease using radiological evaluation techniques in a patient with sigmoid volvulus. In the same consensus, it was decided that barium enema is not necessary to confirm the diagnosis of Hirschsprung's disease, but it aids in the determination of the extent of involved aganglionic bowel segment to guide the operative strategy (37).

On rectal examination, a massive discharge of flatus and stool is expected in Hirschsprung's disease, but this may not be observed when sigmoid volvulus is present. Most patients (97\%) in this study were younger than 40 years, which suggests that Hirschsprung's disease should be suspected in patients younger than 40 years with cases of sigmoid volvulus (Figure 2). Previously published guidelines from Salas et al. (3) on the management of sigmoid volvulus with suspected Hirschsprung's disease do not mention the aspects of age or colon viability. Therefore, we recommend that in patients who have signs of suspected Hirschsprung's disease (complaints of constipation since childhood and patients younger than 40 years) at the time of sigmoid volvulus diagnosis, rectal biopsy should be performed. This is more likely to be recommended if the patient is male.

During the evaluation of patients with sigmoid volvulus together with megacolon, physicians should consider not only Hirschsprung's disease but also degenerative myopathies, such as Bantu's pseudo-Hirschsprung's disease or isolated hypoganglionosis. Degenerative myopathies are inherited diseases, such as Hirschsprung's disease, and there are also adult forms of the disease that can be seen in patients in the age range of 30 to 34 years. In these patients, diagnoses can also be made by rectal biopsy (38-41).

In patients with sigmoid volvulus with suspected Hirschsprung's disease, rectal biopsy is needed before sigmoid resection. Definitive surgery should be planned according to the result of the rectal biopsy. In the first stage, detorsion should be performed by endoscopic, enema, or surgical means. When Hirschsprung's disease is excluded by an intraoperative frozen biopsy, the surgeon can continue definitive surgery. In this study, only 4 of the 21 patients (19.0\%) were diagnosed through an intraoperative frozen biopsy. A colostomy was performed, and definitive surgery was performed in the second session in 3 patients. In the remaining 1 patient, owing to the presence of gangrene in the entire colon, total colectomy with J pouch ileoanal anastomosis was performed in the initial operation.

In this study, the incidence of sigmoid gangrene was lower in patients with sigmoid volvulus with Hirschsprung's disease (9.7\%). We think that the presence of gangrene is not an obstacle to rectal biopsy. In these patients, resection is essential and this colon resection can make definitive surgery more difficult. Moreover, 3 cases of patients with gangrene were reported in the literature, and only 1 of these patients underwent rectal biopsy. The Hartmann's procedure was performed in a 12-year-old male patient with gangrenous sigmoid volvulus (17). A 38-year-old male patient with gangrenous sigmoid volvulus was diagnosed as having a gangrenous sigmoid colon extending to the transverse and ascending colon. This patient underwent proctocolectomy with ileal pouch-anal anastomosis (19). An 82-year-old man was initially followed up conservatively. Laparotomy was performed owing to evolving acute abdominal findings. The Hartmann's procedure was performed because a gangrenous sigmoid colon was observed during the exploration (26).

When Hirschsprung's disease is suspected in patients with sigmoid volvulus, colostomy as the first-step surgical procedure is more appropriate if a sigmoid resection is necessary. If the patient has overlooked Hirschsprung's disease, the risk of anastomotic leakage is high owing to the distal aganglionic segment. If there is suspicion of Hirschsprung's disease in a patient and anastomosis was performed, rectal tube placement may be recommended.

The limitations of this review are that most studies included in this review were case reports and the operations performed for Hirschsprung's disease were heterogeneous. Sigmoid volvulus association with Hirschsprung's disease is a rare event. Therefore, 
this review is not appropriate for meta-analysis, or inclusion of randomized controlled trials is needed to make it appropriate. Although there is a low risk of bias for the included studies, this review suggests a low-quality recommendation, but we think that the recommendations are easily applicable and will assist in avoiding mortality and morbidity.

In patients with constipation since childhood, especially in those with sigmoid volvulus who are younger than 40 years, the likelihood of an underlying Hirschsprung's disease should be considered, and this diagnosis should be excluded before resection and anastomosis of the colon.

Ethics Committee Approval: Since it was a systematic review, its approval to the ethics committee was not required. However, this systematic review was registered in an international database (PROSPERO, CRD42020166692).

Patient Consent for Publication: N/A.

Author Contributions: Concept - U.U.; Design - U.U., C.K.; Supervision - C.K.; Resources - U.U., Ö.G.; Materials - U.U., Ö.G.; Data Collection and/or Processing - U.U., C.K.; Analysis and/or Interpretation - U.U., C.K.; Literature Search - U.U., C.K.; Writing Manuscript - U.U., C.K.; Critical Review - C.K.

\section{Data-sharing Statement: N/A.}

Conflict of Interest: The authors have no conflicts of interest to declare.

Funding: The authors declared that this study has received no financial support.

\section{REFERENCES}

1. Puri P. Hirschsprung's Disease. In: Puri P, editor. Newborn Surgery. London: Arnold; 2003. p.513-33. [Crossref]

2. Miyamoto M, Egami K, Maeda S, Ohkawa K, Tanaka N, Uchida E, et al. Hirschsprung's Disease in Adults: Report of A Case and Review of the literature. J Nippon Med Sch 2005;72:113-20. [Crossref]

3. Salas S, Angel CA, Salas N, Murillo C, Swischuk L. Sigmoid Volvulus in Children and Adolescents. J Am Coll Surg 2000;190:717-23. [Crossref]

4. Huang WK, Li XL, Zhang J, Zhang SC. Prevalence, Risk Factors, and Prognosis of Postoperative Complications after Surgery for Hirschsprung Disease. J Gastrointest Surg 2018;22:335-43. [Crossref]

5. Atamanalp SS. Treatment of Sigmoid Volvulus: A Single-Center Experience of 952 Patients Over 46.5 Years. Tech Coloproctol 2013;17:561-9. [Crossref]

6. Lou Z, Yu ED, Zhang W, Meng RG, Hao LQ, Fu CG. Appropriate Treatment of Acute Sigmoid Volvulus in the Emergency Setting. World J Gastroenterol 2013;19:4979-83. [Crossref]

7. Shea BJ, Reeves BC, Wells G, Thuku M, Hamel C, Moran J, et al. AMSTAR 2: A Critical Appraisal Tool for Systematic Reviews that Include Randomised or Non-Randomised Studies of Healthcare Interventions, or Both. BMJ 2017;358:j4008. [Crossref]

8. Higgins JPT, Green S (editors). Cochrane Handbook for Systematic Reviews of Interventions Version 5.1.0 [updated March 2011]. The Cochrane Collaboration, 2011. Available from www.handbook.cochrane.org.

9. Dean GO, Murry JW. Volvulus of the Sigmoid Colon. Ann Surg 1952;135:830-40. [Crossref]

10. Zeng M, Amodio J, Schwarz S, Garrow E, Xu J, Rabinowitz SS. Hirschsprung Disease Presenting as Sigmoid Volvulus: A Case Report and Review of the Literature. J Pediatr Surg 2013;48:243-6. [Crossref]

11. Buts JP, Claus D, Beguin JC, Otte JB. Acute and Chronic Sigmoid Volvulus in Childhood: Report of Three Cases. Z Kinderchir 1980;29:29-36. [Crossref]

12. McCalla TH, Arensman RM, Falterman KW. Sigmoid Volvulus in Children. Am Surg 1985;51:514-9.

13. Erdener A, Ulman I, Ozcan C, Genc K. A Case of Sigmoid Volvulus Secondary to Hirschsprung's Disease. Pediatr Surg Int 1995;10:409-10. [Crossref]
14. Venugopal KS, Wilcox DT, Bruce J. Hirschsprung's Disease Presenting as Sigmoid Volvulus in A Newborn. Eur J Pediatr Surg 1997;7:172-3. [Crossref]

15. Sarığlu A, Tanyel FC, Büyükpamukçu N, Hiçsönmez A. Colonic Volvulus: A Rare Presentation Of Hirschsprung's Disease. J Pediatr Surg 1997;32:117-8. [Crossref]

16. Rachid K, Jaafar A, Mdaghri J, El Mansari O, Ouanani M, Echarrab M, et al. Hirschsprung's Disease in An Adult Presenting as A Sigmoid Volvulus. Lyon Chir 1997;93:193-4.

17. Puneet, Khanna R, Gangopadhyay AN, Shahoo SP, Khanna AK. Sigmoid Volvulus in Childhood: Report of Six Cases. Pediatr Surg Int 2000;16:132-3. [Crossref]

18. Bach O, Rudloff U, Post S. Modification of Mesosigmoidoplasty for Nongangrenous Sigmoid Volvulus. World J Surg 2003;27:1329-32. [Crossref]

19. Tan FL, Tan YM, Heah SM, Seow-Choen F. Adult Hirschsprung's Disease Presenting as Sigmoid Volvulus: A Case Report and Review of Literature. Tech Coloproctol 2006;10:245-8. [Crossref]

20. Ghaemi M, Bahar MM, Motie MR, Hiradfar M, Soltani E, Saremi E. Late Presentation of Hirschsprung's Disease as Sigmoid Colon Volvulus: Report of Four Cases and Review of the Literature. Colorectal Dis 2010;12:704-5. [Crossref]

21. Alagumuthu M, Jagdish S, Kadambari D. Hirschsprung's Disease in Adults Presenting as Sigmoid Volvulus: A Report of Three Cases. Trop Gastroenterol 2011;32:343-5.

22. Ibrahim I, Mohamed Osman. Transcolostomy-site endorectal pullthrough for Hirschsprung's disease. Ann Pediatr Surg 2013;9:11-5. [Crossref]

23. Khalayleh H, Koplewitz BZ, Kapuller V, Armon Y, Abu-Leil S, Arbell D. Neonatal Sigmoid Volvulus. J Pediatr Surg 2016;51:1782-5. [Crossref]

24. Ranjan A, Jain V, Sharma S, Gupta DK. Sigmoid Volvulus: An Uncommon Complication of Hirschsprung's Disease. BMJ Case Rep 2016: bcr2016214693. [Crossref]

25. Von Horebeek I, Hoffman I, Witters P. A Coffee Bean in An Infant: Call the Endoscopist. J Paediatr Child Health 2017;53:516. [Crossref]

26. Wu S, Sun X, Yu Y, Shen Y. Hirschsprung's Disease-Related Giant Sigmoid Volvulus Complicated by Refractory Hypertension in an Elderly Man. Am J Case Rep 2018;19:467-71. [Crossref]

27. Badner JA, Sieber WK, Garver KL, Chakravarti A. A Genetic Study of Hirschsprung Disease. Am J Hum Genet 1990;46:568-80.

28. Parisi MA, Kapur RP. Genetics of Hirschsprung Disease. Curr Opin Pediatr 2000;12:610-7. [Crossref]

29. Torfs C. An epidemiological study of Hirschsprung disease in a multiracial California population. Evian, France: The Third International Meeting: Hirschsprung Disease and Related Neurocristopathies; 1998.

30. Langer JC. Hirschsprung Disease. In: Holcomb GW 3rd, Murphy JP, Ostlie DJ, editors. Ashcraft's pediatric surgery. 6th edition. Philadelphia: Saunders Elsevier; 2010. p.474-91.

31. Ikeda K, Goto S. Diagnosis and Treatment of Hirschsprung's Disease in Japan. An Analysis of 1628 Patients. Ann Surg 1984;199:400-5. [Crossref]

32. Fairgrieve J. Hirschsprung's Disease in the Adult. Br J Surg 1963;50:506-14. [Crossref]

33. McCready RA, Beart RW Jr. Adult Hirschsprung's Disease: Results of the Surgical Treatment at Mayo Clinic. Dis Colon Rectum 1980;23:401-7. [Crossref]

34. Barnes PR, Lennard-Jones JE, Howley PR, Todd IP. Hirschsprung's Disease and Idiopathic Megacolon in Adults and Adolescents. Gut 1986;27:534-41. [Crossref]

35. Rich AJ, Lennard TW, Wilsdon JB. Hirschsprung's Disease as A Cause of Chronic Constipation in the Elderly. Br Med J (Clin Res Ed) 1983;287:1777-8. [Crossref]

36. Burki T, Sinha CK, Yamataka A. Hirschsprung's Disease. In: Sinha C, Davenport M (eds) Handbook of Pediatric Surgery. Springer, London. 2010; p. 117-24. [Crossref]

37. Martucciello G, Pini Prato A, Puri P, Holschneider AM, Meier-Ruge W, Jasonni V, et al. Controversies Concerning Diagnostic guidelines for Anomalies of the Enteric Nervous System: A Report from the Fourth International Symposium on Hirschsprung's Disease and Related Neurocristopathies. J Pediatr Surg 2005;40:1527-31. [Crossref]

38. Qadir I, Salick MM, Barakzai A, Zafar H. Isolated adult Hypoganglionosis Presenting as Sigmoid Volvulus: A Case Report. J Med Case Rep 2011;5:445. [Crossref]

39. Gabbani T, Marsico M, Marocchi M, Biagini MR. Isolated Hypoganglionosis in Young Man with Autism. Dig Liver Dis 2017;49:104. [Crossref]

40. Rode H, Moore SW, Kaschula ROC, Brown RA, Cywes S. Degenerative Leiomyopathy in Children. A Clinico-Pathological Study. Pediatr Surg Int 1992;7:23-29. [Crossref]

41. Katz A. Pseudo-Hirschsprung's Disease in Bantu Children. Arch Dis Child 1996;41:152. 